\title{
Myocardial insulin resistance does not always parallel skeletal muscle and whole body insulin resistance: A mini review
}

\author{
—Myocardial Insulin Resistance
}

\author{
Ikuo Yokoyama \\ Department of Cardiovascular Medicine and Preventive Medicine, Clinical Research Institutes of Sanno Hospital and Sanno Medical \\ Center, International University of Health and Welfare, Tokyo, Japan \\ Email: yokochan-tky@umin.ac.jp
}

Received 16 November 2012; revised 20 December 2012; accepted 30 December 2012

\begin{abstract}
Insulin resistance (IR) is recognized to be of critical importance in a variety of metabolic diseases and coronary artery disease (CAD). Impaired skeletal muscle glucose utilization (SMGU) plays an important role in the pathogenesis of IR, whereas it is controversial whether myocardial IR is similar in this respect. Methods: Twenty-two studies of myocardial IR and skeletal muscle IR using positron emission tomography (PET) and/or whole body IR were reviewed. Heart and skeletal muscle IR were measured with PET and ${ }^{18}$ F-FDG under hyperinsulinemic euglycemic insulin clamp technique. Whole body IR was also determined at the time of PET under hyperinsulinemic euglycemic insulin clamp technique. Results: One study reported that heart and skeletal muscle IR is present in untreated type 2 diabetes mellitus (T2DM), hypertension and CAD (as reflected in a myocardial glucose utiliation rate (MGU) in T2DM vs control [p $<0.01$ ], and an SMGU in T2DM vs control [p $<0.01])$. A significant negative relationship between MGU and FFA $(\mathrm{r}=-\mathbf{0 . 6 6 5}, \mathrm{p}<0.01)$ and a significant positive relationship between MGU and whole body IR $(r=0.855$, $p<0.01)$ was also observed in T2DM. Significantly reduced MGU and SMGU and a positive correlation between them $(r=0.78, p<0.0001)$ were noted in the normal myocardial segments of patients with CAD. Another study showed that heart and skeletal muscle IR was present in T2DM both with CAD (MGU): p < 0.01; SMGU: $p<0.01$ ) or without CAD (MGU: $p<$ 0.01 ; SMGU: $p=0.06$ ). A significant positive relationship between the whole body glucose disposal rate and MGU $(r=0.60, p<0.01)$ as well as $\operatorname{SMGU}(r=0.76, p$ $<0.01)$ was also reported. Much more severe myocardial IR in T2DM and hypertriglyceridemia $(p<0.05)$ due to increased plasma free fatty acids, FFA ( $r=$
\end{abstract}

$-0.60, p<0.01)$ and plasma triglycerides levels $(r=$ $-0.74, p<0.001$ ) was reported. A significant negative relationship between MGU and plasma triglycerides ( $r$ $=-0.74, p<0.001)$ was also noted. However, other studies reported that MGU was increased in essential hypertension despite the fact that SMGU and whole body IR were present. Furthermore, it has also been reported that MGU in hypertensive T2DM without medication for diabetes was similar to controls $(p=n s)$ despite the presence of skeletal muscle IR $(p<0.01)$ and whole body IR. Myocardial IR was not detected in non-diabetic non-hypertensive hypertriglyceridemia (Myocardial ${ }^{18}$ F-FDG Uptake (MFU) in hypertriglyceridemics was $(p=n s)$ despite findings of reduced skeletal muscle ${ }^{18}$ F-FDG uptake (SMFU in hypertriglyceridemia $(p<0.01)$ and whole body IR (GDR) in hypertriglyceridemia $(p<0.01)$. Myocardial IR was not detected under very high dose insulin clamping (about 10 times higher than the usual dose) with MGU in T2DM vs control $p=n s$, while whole body IR in T2DM was still present, $(p<0.02)$ and skeletal muscle $(p<0.01)$. Preserved MGU under chronic use of sulfonylurea drugs in T2DM has been reported (T2DM with SU vs control, $p=n s)$. Moreover, myocardial IR was not seen in hypertensive T2DM despite the existence of skeletal muscle and whole body IR. Myocardial IR in T2DM with CAD could be improved by thiazolidinediones (MGU before rosiglitazone, vs after rosiglitazone $p<0.05$ ) and in mixed combined hyperlipidemia with CAD before-vs-after pioglitazone ( $p<$ 0.01). This suggests that myocardial IR in T2DM and CAD paralleled an improvement of skeletal muscle and whole body IR. However, troglitazone failed to improve myocardial IR in T2DM without CAD within 12 weeks therapy (MGU before therapy vs after, $p=$ ns). Nonetheless, it did improve myocardial IR in 
T2DM without CAD after 12 months' therapy (before therapy vs after 12 months, $p<0.05$ ). Conclusion: The myocardium possesses mechanisms to resist IR different from those in the rest of the body. Therefore, myocardial IR does not always parallel skeletal muscle and whole body IR.

Keywords: Insulin Resistance; Myocardial Insulin Resistance; Coronary Artery Disease; Metabolic Syndrome; Diabetes; Hyperlipidemia; PET; FDG

\section{INTRODUCTION}

Life-styles in Japan are changing from the traditional to the Western, associated with increasing occurrence of diabetes mellitus, hyperlipidemia and metabolic syndrome [1], leading to increased future risks of cardiovascular events. Metabolic syndrome has emerged as a new concept which is charactzerized by a cluster of metabolic and coronary factors leading to an increased risk of coronary artery disease (CAD) [2]. A report from the Ministry of Health and Welfare of Japan has shown that the percentage of middle-aged men and women with metabolic syndrome in the country is now around 50\% and $20 \%$, respectively. It is also reported that the incidence of metabolic syndrome is still increasing in Western countries [3]. Therefore, this condition is becoming a major health concern both in Western countries and Japan. Because it includes abdominal obesity, essential hypertension, glucose intolerance and hypertriglyceridemia, in which insulin resistance plays a central role, investigations of the latter are crucial. Insulin resistance is defined as an impaired glucose utilization response to the stimulatory effect of insulin and has been recognized to be very important in many diseases. Because skeletal muscle glucose consumption accounts for approximately $80 \%$ of total glucose utilization in the whole body, an impaired skeletal muscle glucose utilization rate (SMGU) is thought to play a central role in the pathogenesis of insulin resistance. However, role of myocardial insulin resistance on the whole body insulin resistance has been remain uncertain.

Positron emission tomography (PET) has emerged as a new clinical diagnostic tool that allows non-invasive quantitative analysis in several human tissue metabolisms and blood flow in vivo. In principle, PET has the ability to determine absolute value of heart and skeletal muscle glucose utilization rate, and its use might facilitate investigations of the pathophysiology of both heart diseases and metabolic diseases. PET could also be valuable in assessing the effects of certain drugs on heart and skeletal muscle glucose utilization or myocardial vasodilatory functions and for developing new drugs associated with coronary risk factors and/or metabolic syndrome. Therefore, this non-invasive technique could also offer valuable insights into insulin resistance in clinical settings via its ability to quantify SMGU [4,5] and the myocardial glucose utilization rate [MGU] [6]. Recently, Yokoyama et al. reported that SMGU could be measured as a ratio of SUV or skeletal muscle to blood ${ }^{18}$ FDG count ratio simply on the basis of static skeletal muscle PET imaging [4]. They also reported that sequential dynamic PET scans of the thoracic and femoral regions could determine both MGU and femoral muscle SMGU in little more than one hour, without the requirement for arterial blood sampling [5]. This suggested that heart and skeletal muscle glucose utilization rates could be obtained routinely from only one PET scan, obviating the need for arterial blood sampling. ${ }^{18}$ F-FDG PET has been used extensively in research on quantitative analysis of heart and skeletal muscle insulin resistance using hyperinsulinemic euglycemic glucose clamp technique. Thus in this manuscript, I review clinical studies of heart and skeletal muscle insulin resistance using PET and ${ }^{18} \mathrm{~F}$-FDG to explore whether similarities and differences in insulin resistance between heart and skeletal muscle could exist.

\section{MAIN PART OF REVIEW AND DISCUSSION}

Based on the methods to measure the skeletal muscle glucose utilization rate using PET and ${ }^{18}$ F-FDG and hyperinsulinemic euglycemic glucose clamp technique [4-6], there have been several clinical reports that investtigated the insulin resistance in the skeletal muscle in several diseases and it's relationship to whole body insulin resistance using PET and ${ }^{18}$ F-FDG and hyperinsulinemic euglycemic glucose clamp technique. Reduced SMGU during insulin clamping, implying insulin resistance in the skeletal muscle, has been reported in patients with type-2 diabetes (T2DM) [7,8], patients with hypertension [9] and patients with non-diabetic non-hypertensive hypertriglyceridemia [10] using quantitative PET and ${ }^{18} \mathrm{~F}$-FDG and and hyperinsulinemic euglycemic glucose clamp technique. Reduced SMGU and linear positive relationship between SMGU and whole body glucose disposal rate during insulin claming was also shown in patients with T2DM, non-diabetic hypertension and non-diabetic non-hypertensive hypertriglyceridemia with similar magnitude [11]. Furthermore, it has also been reported that SMGU in T2DM can be improved by the insulin sensitizer "troglitazone" which acts as a PPAR- $\gamma$ agonist with or without association of essential hypertension [12]. Peltoniemi et al. have shown that both SMGU at rest and after its exercise-induced increase was lower in patients with type 1 diabetes (T1DM) relative to healthy controls, suggesting that skeletal muscle insulin 
resistance is also present even in patients with T1DM. These results imply that exercise therapy may be effecttive for T1DM treatment, but still has some limitations [13]. In addition, an existence of such skeletal muscle insulin resistance in T1DM has indicated that hyperglycemia could be one of factor to provide insulin resistance.

On the other hands, there have been several discussions as to whether myocardial insulin resistance actually exists and if so, whether it is similar to skeletal muscle insulin resistance? Cardiac muscle preferentially utilizes glucose as the primary energy source under conditions of nutrient repletion, while utilizing free fatty acids (FFA) as predominat source of energy under fasting conditions. Furthermore, glucose is an important substrate for maintenance of normal cardiac function and viability in the ischemic heart, whereby insulin resistance plays an essential role in the development of CAD. In fact, Nuutila et al. observed that MGU and SMGU were reduced in normal subjects following infusion of intralipid and the associated increase in FFA. This finding strongly suggested that a glucose-FFA cycle is in operation in heart and skeletal muscle in normal humans [14]. In addition, heart and skeletal muscle insulin resistance has also been reported in patients with T2DM without CAD [7]. Yokoyama et al. also showed that heart and skeletal muscle insulin resistance was present in T2DM patients not on medication, and without essential hypertension and CAD [8]. They also observed a significant negative relationship between MGU and plasma FFA concentrations, and a significant positive association between MGU and whole body glucose disposal rate (which is a marker of insulin resistance in T2DM). This also supported the existence of a glucose-FFA cycle in heart and skeletal muscle in patients with T2DM. It is also reported that myocardial insulin resistance may occur in normal myocardial segments of patients with CAD [15]. In that study, significantly reduced MGU and SMGU was observed, and a positive association between SMGU and MGU was documented. Another study has also shown that heart and skeletal muscle insulin resistance can be present in T2DM patients whether or not they have CAD [16]. These investigators also observed a significant positive association between SMGU and MGU. Much more severe myocardial insulin resistance in patients with T2DM and severe hypertriglyceridemia due to increased plasma FFA levels was also reported [17]. A significant negative correlation between MGU and plasma triglycerides levels was observed. These data have been taken to suggest that myocardial insulin resistance does exist and is similar to skeletal muscle insulin resistance. On the other hand, other data imply that myocardial insulin resistance does not always parallel skeletal mus- cle insulin resistance. For example, MGU was reported to be increased in essential hypertension while SMGU and the whole body glucose disposal rate were significantly reduced in the same study patients [9]. Furthermore, Yokoyama et al. also reported that MGU was similar in both hypertensive T2DM and controls despite significantly reduced SMGU and whole body glucose disposal rates in the former indicating an existence of insulin resisatnce in skeletal muscle and whole body but not in the heart in patients with T2DM and hypertension [18]. Moreover, myocardial insulin resistance was not observed when using very high insulin doses for insulin clamping (about 10 times higher than usual) while insulin resistance in the whole body and skeletal muscle was still present [19]. Consistent with this discrepancy, preserved MGU in response to insulin clamping under chronic use of sulfonylurea drugs in T2DM has been also reported [20]. In contrast to the fact that skeletal muscle insulin resistance is present in type 1 diabetes, myocardial insulin resistance was not observed [21]. Preserved MGU but reduced SMGU under insulin clamping was also shown in patients with hypertriglyceridemia despite the apparent existence of whole body insulin resistance [10]. Therefore, the myocardium may have alternative so-far unidentified mechanisms to oppose insulin resistance (Figure 1). Moreover, using ${ }^{18}$ F-FDG PET under insulin clamping to study the effects of thiazolidinediones which act as insulin sensitizers on the impaired heart and skeletal muscle glucose utilization in T2DM, has also yielded data supporting this notion. Using a randomized placebo-controlled study design, Lautamaeki et al. recently reported that 16 -week rosiglitazone (one of thiazolidinediones) therapy improved both heart and skeletal muscle glucose utilization in T2DM with CAD

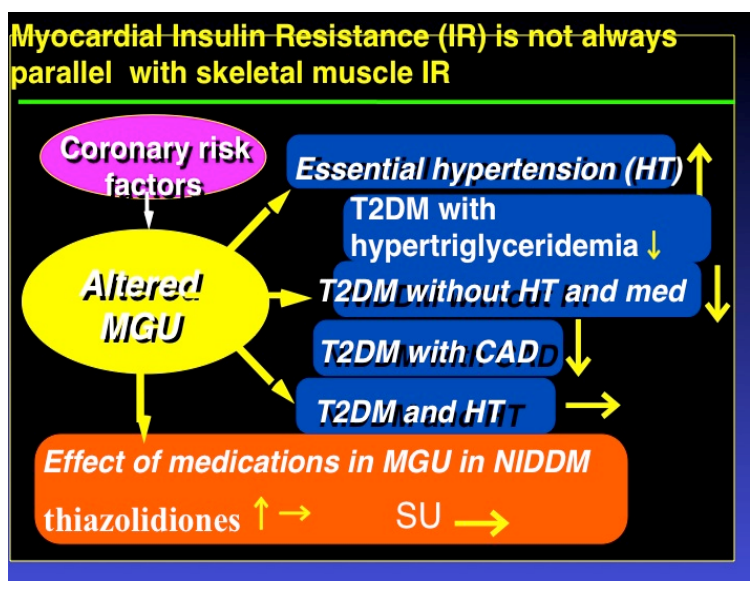

Figure 1. Complicated myocardial insulin resistance and nor always parallel Skeletal Muscle and Whole Body Insulin Resistance; T2DM: type-2 diabetes, CAD: coronary artery disease, SU: sulfonylurea drugs, MGU: myocardial glucose utilization rate. 
[22]. Because thiazolidinediones such as pioglitazone and rosiglitazone act as insulin sensitizers, improvement of MGU in T2DM by these drugs might indicate the occurrence of myocardial insulin resistance in patients with T2DM. Similar results using pioglitazone were obtained by Naoumova et al. [23].

However, Yokoyama et al. showed that heart muscle glucose utilization in patients with T2DM without CAD could not be improved within 12 weeks by an acute use of troglitazone [24]. However, they did show that troglitazone improved heart and skeletal muscle insulin resistance over 12 months' therapy [24]. Delayed troglitazone responses before an improvement of myocardial insulin resistance is observed suggests that mechansisms for improving myocardial insulin resistance could differ in these three thiazolidiones, although all of them (troglitazone, pioglitazone, rosiglitazone) do improve whole body and skeletal muscle insulin resistance to almost the same extent. In contrast, both of two insulin sensitizers, troglitazone and pioglitazone failed to improve myocardial flow reserve. Whereas recent report by yokoyama et $a l$. have revealed that intensive control of hyperglycemia could provide recovery of coronary microangiopathy in patients with T2DM [25]. These results are in good agreement with our previously reported that results that myocardial microvascular dysfunction in response to dipyridamole as expressed by a reduced myocardial flow reserve in T2DM is not related to whole body insulin resistance [26].

\section{CONCLUSION}

In conclusion, it seems that the myocardium has alternative mechanisms for managing insulin resistance and that myocardial insulin resistance does not always parallel skeletal muscle and whole body insulin resistance.

\section{REFERENCES}

[1] Ueshima, K., Oba, K., Yasuno, S., Fujimoto, A., Tanaka, S., Ogihara, T., Saruta, T. and Nakao, K. (2011) Influence of coronary risk factors on coronary events in japanese high-risk hypertensive patients. Primary and secondary prevention of ischemic heart disease in a subanalysis of the candesartan antihypertensive survival evaluation in Japan (CASE-J) trial. Circulation Journal, 75, 24112416. doi:10.1253/circj.CJ-10-1161

[2] Steven, M. and Haffner, M.D. (2006) Risk constellations in patients with the metabolic syndrome: Epidemiology, diagnosis, and treatment patterns. The American Journal of Medicine, 119, S3-S9. doi:10.1016/j.amjmed.2006.01.008

[3] Steven, M. and Haffner, MD. (2006) Relationship of metabolic risk factors and development of cardiovascular disease and diabetes. Obesity, 14, 121S-127S. doi:10.1038/oby.2006.291
[4] Yokoyama, I., Inoue, Y., Moritan, T., Ohtomo, K. and Nagai, R. (2003) Simple quantification of skeletal muscle glucose utilization by static 18F-FDG PET. Journal of Nuclear Medicine, 44, 1592-1598. doi:10.1097/00006231-200501000-00006

[5] Yokoyama, I., Inoue, Y., Moritan, T., Ohtomo, K. and Nagai, R. (2005) Measurement of skeletal muscle glucose utilization by dynamic 18F-FDG PET without arteial blood sampling. Nuclear Medicine Communications, 26, 31-37.

[6] Ohtake, T., Kosaka, N., Watanabe, T., Yokoyama, I., Moritan, T., Masuo, M., Iizuka, M., Kozeni, K., Momose, T., Oku, S., Nishikawa, J., Sasaki, Y. and Iio, M. (1991) Noninvasive method to obtain input function for measuring tissue glucose utilization of thoracic and abdominal organs. Journal of Nuclear Medicine, 32, 1432-1438.

[7] Voipio-Pulkki, L.M., Nuutila, P., Knuuti, M.J., Ruotsalainen, U., Haaparanta, M., Teräs, M., Wegelius, U. and Koivisto, V.A. (1993) Heart and skeletal muscle glucose disposal in type 2 diabetic patients as determined by positron emission tomography. Journal of Nuclear Medicine, 34, 2064-2067.

[8] Yokoyama, I., Yonekura, K., Ohtake, T., Kawamura, H., Matsumoto, A., Inoue, Y., Aoyagi, T., Sugiura, S., Omata, M., Ohtomo, K. and Nagai, R. (2000) Effect of insulin resistance on Heart and skeletal muscle FDG uptake in type II diabetics. Journal of Nuclear Cardiology, 7, 242248. doi:10.1016/S1071-3581(00)70013-4

[9] Nuutila, P., Mäki, M., Laine, H., Knuuti, M.J., Ruotsalainen, U., Luotolahti, M., Haaparanta, M., Solin, O., Jula, A., Koivisto, V.A., et al. (1995) Insulin action on heart and skeletal muscle glucose uptake in essential hypertension. Journal of Clinical Investigation, 96, 10031009. doi:10.1172/JCI118085

[10] Yokoyama, I., Ohtake, T., Momomura, S., Yonekura, K., Kobayakawa, N., Aoyagi, T., Sugiura, S., Yamada, N., Ohtomo, K., Sasaki, Y., Omata, M. and Yazaki, Y. (1999) Insulin action on Heart and skeletal muscle FDG uptake in patients with hypertriglyceridemia. Journal of Nuclear Medicine, 40, 1116-1121.

[11] Yokoyama, I., Moritan, T. and Inoue, Y. (2012) Functional imaging of skeletal muscle glucose metabolism by 18FDG PET to characterize insulin resistance in patients at high risk for coronary artery disease. Journal of Biomedical Science and Engineering, 5, 819-825. doi:10.4236/jbise.2012.512A103

[12] Yokoyama, I., Yonekura, K., Moritan, T., Tateno, M., Momose, T., Ohtomo, K., Inoue, Y. and Nagai, R. (2001) Troglitazone can improve impaired femoral muscle glucose utilization in type II diabetics with or without hypertension. Journal of Nuclear Medicine, 42, 1005-1010.

[13] Peltoniemi, P., Yki-Järvinen, H., Oikonen, V., Oksanen, A., Takala, T.O., Rönnemaa, T., Erkinjuntti, M., Knuuti, M.J. and Nuutila, P. (2001) Resistance to exercise-induced increase in glucose uptake during hyperinsulinemia in insulin-resistant skeletal muscle of patients with type 1 diabetes. Diabetes, 50, 1371-1377. doi:10.2337/diabetes.50.6.1371

[14] Nuutila, P., Koivisto, V.A., Knuuti, J., Ruotsalainen, U., 
Teräs, M., Haaparanta, M., Bergman, J., Solin, O., VoipioPulkki, L.M., Wegelius, U., et al. (1992) Glucose-free fatty acid cycle operates in human heart and skeletal muscle in vivo. Journal of Clinical Investigation, 89, 1757-1744. doi:10.1172/JCI115780

[15] Paternostro, G., Camici, P.G., Lammerstma, A.A., Marinho, N., Baliga, R.R., Kooner, J.S., Radda, G.K. and Ferrannini, E. (1996) Cardiac and skeletal muscle insulin resistance in patients with coronary heart disease a study with positron emission tomography. Journal of Clinical Investigation, 98, 2094-2099. doi:10.1172/JCI119015

[16] Iozzo, P., Chareonthaitawee, P., Dutka, D., Betteridge, D.J., Ferrannini, E. and Camici, P.G. (2002) Independent association of type 2 diabetes and coronary artery disease with myocardial insulin resistance. Diabetes, 51, 30203024. doi:10.2337/diabetes.51.10.3020

[17] Monti, L.D., Landoni, C., Setola, E., Galluccio, E., Lucotti, P., Sandoli, E.P., Origgi, A., Lucignani, G., Piatti, P. and Fazio, F. (2004) Myocardial insulin resistance associated with chronic hypertriglyceridemia and increased FFA levels in type 2 diabtic patients. American Journal of Physiology-Heart and Circulatory Physiology, 287, H1225-H1231. doi:10.1152/ajpheart.00629.2003

[18] Yokoyama, I., Ohtake, T., Momomura, S., Yonekura, K., Yamada, N., Nishikawa, J., Sasaki, Y. and Omata, M. (1998) Organ specific insulin resistance in patients with non-insulin dependent diabetes mellitus and hypertension. Journal of Nuclear Medicine, 39, 884-889.

[19] Utriainen, T., Takala, T., Luotolahti, M., Rönnemaa, T., Laine, H., Ruotsalainen, U., Haaparanta, M., Nuutila, P. and Yki-Järvinen, H. (1998) Insulin resistance characterizes glucose uptake in skeletal muscle but not in the heart in NIDDM. Diabetologia, 41, 555-559. doi:10.1007/s001250050946

[20] Yokoyama, I., Inoue, Y., Moritan, T., Ohtomo, K. and Nagai, R. (2006) Myocardial glucose utilization in type II diabetes mellitus patients treated with sulphonylurea drugs. European Journal of Nuclear Medicine and Molecular, 33, 703-708. doi:10.1007/s00259-005-0042-x
[21] Nuutila, P., Knuuti, J., Ruotsalainen, U., Koivisto, V.A., Eronen, E., Teräs, M., Bergman, J., Haaparanta, M., Voipio-Pulkki, L.M., Viikari, J. et al. (1993) Insulin resistance is localized to skeletal but not heart muscle in type 1 diabetes. American Journal of Physiology, 264, E756-E762.

[22] Lautamaki, R., Airaksinen, K.E., Seppanen, M., Toikka, J., Luotolahti, M., Ball, E., Borra, R., Harkonen, R., Iozzo, P., Stewart, M., Knuuti, J. and Nuutila, P. (2005) Rosiglitazone improves myocardial glucose uptake in patients with type 2 diabetes and coronary artery disease a 16-week randomized, double-blind, placebo-controlled study. Diabetes, 54, 2787-2794. doi:10.2337/diabetes.54.9.2787

[23] Naoumova, R.P., Kindler, H., Leccisotti, L., Mongillo, M., Khan, M.T., Neuwirth, C., Seed, M., Holvoet, P., Betteridge, J. and Camici, P.G. (2007) Pioglitazone improves myocardial blood flow and glucose utilization in nondiabetic patients with combined hyperlipidemia. A randomized, double-blind, placebo-controlled study. Journal of the American College of Cardiology, 50, 20512058. doi:10.1016/j.jacc.2007.07.070

[24] Yokoyama, I., Moritan, T. and Inoue, Y. (2012) Heart and skeletal muscle insulin resistance but not myocardial blood flow reserve could be related to chronic use of thiazolidione in type-2 diabetics. Journal of Biomedical Science and Engineering, 5, 829-835. doi:10.4236/jbise.2012.512A105

[25] Yokoyama, I., Inoue, Y. and Moritan, T. (2012) Recovery of coronary microangiopathy in patients with type 2 diabetes. Journal of Hypo \& Hyperglycemia, in press.

[26] Yokoyama, I., Ohtake, T., Momomura, S., Yonekura, K., Woo-Soo, S., Nishikawa, J., Sasaki, Y. and Omata, M. (1998) Hyperglycemia rather than insulin resistance is related to coronary flow reserve in patients with non-insulin dependent diabetes mellitus. Diabetes, 47, 119-124. doi:10.2337/diabetes.47.1.119 